\title{
Implicit alternatives to a stimulus, difficulty of encoding, and schema-plus-correction representation*
}

\author{
GORDON BEAR \\ University of Wisconsin, Madison, Wisconsin 53706
}

\begin{abstract}
Garner has hypothesized that a S exposed to a stimulus infers alternatives to it. Data are reviewed which establish a close correlation between the number of implicit alternatives to a stimulus and the difficulty of encoding that stimulus. This correlation provides a datum to be explained and suggests that the identification of specific implicit alternatives may provide clues to the encoding process. For dot patterns of a certain kind, it has been suggested that the rotations and reflections of a stimulus are its only alternatives, but a close examination of certain data indicates that, for many of these patterns, the alternatives also include one or more other patterns which differ from the stimulus only in the position of a single dot and which locate that dot in a position where it is more predictable from the other dots. It is suggested that the one-dot-different alternatives represent schemas used in schema-plus-correction encoding.
\end{abstract}

Wendell Garner (1962, Chapter 6, 1966) has argued that a $S$ exposed to a stimulus of some given kind will understand it to be one of a subset of the stimuli of that kind. The other members of the subset, according to Garner's hypothesis, are implicit alternatives to the stimulus-other stimuli which the S understands could have occurred but did not. The present paper reports a review of the data on implicit alternatives, with special attention to a set of dot patterns which Garner and his associates have studied intensively. A variety of measures taken on the dot patterns and certain others provide evidence for a close correlation between the number of implicit alternatives to a stimulus and the difficulty of encoding that stimulus, and attention is directed to this correlation as an important datum to be explained. The correlation also suggests that an effort to identify actual alternatives to particular stimuli may provide some clues to the encoding process, and for the dot patterns in this regard, it has been suggested that the rotations and reflections of a pattern are its only alternatives. A detailed examination of certain data indicates, however, that the alternatives for many of the dot patterns include one or more other patterns which differ from the stimulus only in the position of a single dot and which locate that dot in a position where it is more predictable from the other elements of the stimulus. This finding suggests a type of encoding generally overlooked in current discussions of the process-encoding as a schema plus a correction.

\section{NUMBER OF IMPLICIT ALTERNATIVES AND DIFFICULTY OF ENCODING}

\section{The Dot Patterns}

The stimuli which Garner and his associates have studied so intensively are dot patterns of the kind shown

\footnotetext{
*The author wishes to thank John Flowers for a helpful critique of an earlier draft of this paper.
}

in Fig. 1 (the numbers within the dots have not appeared in the stimuli presented to the Ss, of course). So many data have now been collected on these patterns that they constitute a virtually unique collection of "case histories" in experimental psychology and provide an excellent set of stimuli for further investigation of perceptual and cognitive processes. The patterns consist of five dots distributed over the cells of a 3 by 3 matrix. There are 126 patterns of this kind $\left({ }_{9} \mathrm{C}_{5}\right)$, but certain of them are rotations or reflections of each other. Grouping rotations and reflections together partitions the 126 patterns into 23 mutually exclusive equivalence sets (Prokhovnik, 1959), each set composed of those patterns which are identical to each other if rotated in one or more 90-deg turns, if reflected about the vertical or the horizontal axis, or if both rotated and reflected. Figure 1 displays a representative pattern from each equivalence set. The numeral 1,4 , or 8 in a set's label indicates the size of the set, while the letter in the label distinguishes the set among the several sets of that size. As the figure indicates, there are 2 sets of Size 1, 11 sets of Size 4, and 10 sets of Size 8 .

\section{Number of Implicit Alternatives ${ }^{1}$}

The number of implicit alternatives for a given dot pattern has been measured in three ways. Garner and Clement (1963), first of all, worked with figures lacking the 3 by 3 matrix and thus employed only those 90 patterns with at least one dot in each row and column, so that the boundaries of the figures were suggested to the Ss. The researchers reasoned that the patterns Ss judge to be similar to a stimulus would be representative of those in its subset of implicit alternatives, and they asked their Ss to sort the 90 patterns into approximately eight groups of similar figures. The mean size of the groups into which a figure was placed then served to index the number of its implicit alternatives.

Handel and Garner (1966) later worked with figures drawn to include the matrix and used all 126 of the 

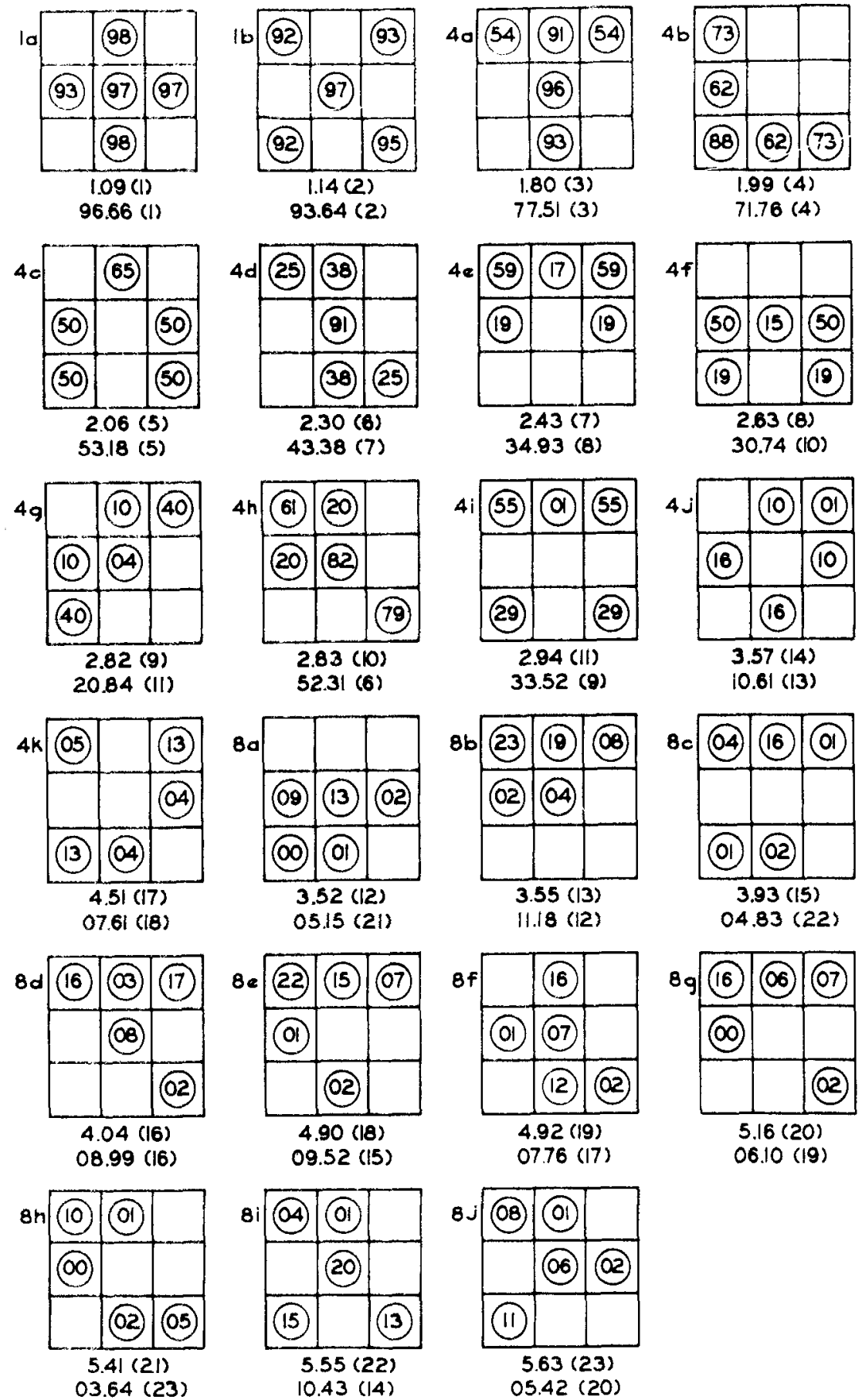

Fig. 1. Data on five-dot patterns. To the left of each pattern, the label for its equivalence set. Below each pattern on the first line, the mean "goodness" rating of the patterns in its set, as determined by Handel and Garner $(1966)-1=\mathrm{a}$ "good" pattern, $7=\mathrm{a}$ "poor" pattern-and the rank of this mean rating. On the second line, the mean of the mean predictability scores for the patterns in the set and the rank of the mean. Within each dot, the mean predictability score for that dot, computed as described in the text. (From Bear, 1973).

patterns. They reasoned that the patterns which Ss associate with a stimulus would be representative of those in its subset of implicit alternatives, and for each of the figures they asked their Ss to draw another one "suggested by" it. Garner and Clement's Ss had been forced to partition the patterns into mutually exclusive subsets, but Handel and Garner obtained a matrix of stimuli and associates which suggested that Ss conceive the patterns to be grouped into a series of nested subsets. The implicit alternatives to a stimulus, those figures that belong to its subset, thus may also belong to one of the smaller groups nested within the subset, it 
appears, but the figures located outside the subset in any larger group which includes the subset do not constitute alternatives to the stimulus. Handel and Garner developed two measures of the number of implicit alternatives for a pattern: the position of the pattern's subset in the nesting hierarchy (a low-numbered position indicates a small inner subset) and the number of times the pattern was used as an associate to other patterns (a greater frequency of use indicates a small inner subset). The correlation between the two measures was - .86 , and they were closely linearly related to Garner and Clement's measure, correlating .90 and -.87 with it. [These coefficients and the others reported below are values for Pearson's r computed by Bear (1970)]. The strong correlations between the measures from the two studies indicate that the inclusion or omission of the nine-cell matrix makes little difference to the Ss, it might be noted.

\section{Difficulty of Encoding}

Difficulty in encoding the dot patterns has been measured in four different ways. Clement asked Ss to generate a one-word name for 50 of the patterns in one study (1964) and to learn to associate a two-digit number with 17 of the patterns in another study (1967). As indices of a pattern's encodability, he computed the median latency and the uncertainty (or variability) of the distribution of names which the Ss supplied for the pattern in the naming task and the mean trials to criterion as a stimulus term and as a response term in the paired-associates task. Correlations among the measures ranged from .74 to .94 , with a median value of .86 . Thus the patterns for which Ss were slow in generating a name tended to be those for which they generally disagreed on the name and those which required many trials to learn as a stimulus term and as a response term.

\section{Correlation Between Number of Alternatives and Difficulty of Encoding}

The patterns which were difficult to encode in Clement's studies prove to be those with many implicit alternatives. The four measures of encoding difficulty and the three measures of number of implicit alternatives yield a matrix of 12 correlations, all in the "right" direction and ranging in absolute value from .61 to .94 , with a median of .80 .

\section{Other Stimuli}

More evidence for a strong correlation between number of implicit alternatives and difficulty of encoding is available in data on other stimuli. Glanzer, Taub, and Murphy (1968) have reported the correlation for dot patterns of a different kind (their data were not completely consistent, however), and Royer and Garner (1966) have reported the correlation for auditory patterns. A variety of other patterns studied by Handel and Buffardi (1968) may also provide evidence for the correlation. Royer and Garner generated sequences of tones by repeating a basic pattern of eight elements, each element one of two sounds; Handel and Buffardi used eight of these auditory sequences plus "translations" of them into eight other modes of presentation. In a visual version, the elements of the patterns were two lights displayed sequentially rather than two tones, and in a tactile version the elements were vibrations applied to the left or the right hand. In three other versions, a basic eight-element pattern was presented first in one modality (auditory, visual, or tactile) and then in a second, and the alternations were continued every eight elements; the three versions here were the three possible pairs of modalities. In another three versions, the elements were a stimulus from one modality (a tone, light, or vibration) and a stimulus from another modality, and again the three versions were all three possible pairs. Handel and Buffardi determined the median number of elements in a sequence that the Ss observed before identifying a pattern, and this score provides a difficulty-of-encoding measure. The rank order of the eight sequences on this measure was little affected by the mode of presentation, by the rate of presentation, and by the point in the basic pattern at which the sequence began, and Handel and Buffardi reported only an overall rank order for difficulty of encoding. This order correlates highly with the ranking of the patterns of the number-of-alternatives measure which Royer and Garner obtained for the stimuli in their auditory version (rho $=.79$; data from Royer and Garner's Table 1 and Handel and Buffardi's Table 1).

In sum, data on a variety of stimuli indicate that a pattern with relatively many implicit alternatives is a pattern which is relatively difficult to encode. This correlation provides a datum which must be explained by any theory of the encoding process.

\section{COMPETITORS AS IMPLICIT ALTERNATIVES}

The correlation between number of implicit alternatives and difficulty of encoding also suggests that the identification of specific alternatives to specific stimuli may provide clues to the encoding process. For the dot patterns in this regard, Clement, following an argument advanced in the Garner-Clement (1963) paper, has asserted that the rotations and reflections of a pattern (the members of its equivalence set) are inferred as alternatives to it (Clement, Guenther, \& Sistrunk, 1972; Clement \& Sistrunk, 1971; Clement, Sistrunk, \& Guenther, 1970; Clement \& Weiman, 1970). There is evidence in the data on number of alternatives that in fact they are. In Garner and Clement's study, which took the patterns judged similar to a stimulus to be representative of its implicit alternatives, the rotations and reflections of a stimulus were generally judged similar to it. In Handel and Garner's study, which took 
the patterns suggested by a stimulus to be representative of its implicit alternatives, the rotations and reflections of a stimulus were generally suggested to the Ss by the stimulus and were used as associates in $39.1 \%$ of the cases-about 10 times as often as would be expected if each pattern had the same chance of being associated to each other pattern.

Clement implies, however, that rotations and reflections are the only implicit alternatives for the dot patterns, and there is evidence to the contrary in Handel and Garner's data. ${ }^{2}$ Their matrix of stimuli and associates indicates that the implicit alternatives for many of the patterns include one or more competitors of the pattern-one or more patterns which differ from the stimulus only in the position of a single dot and in a sense compete with the stimulus for that dot (for example, $1 \mathrm{~b}$ as an alternative to $4 \mathrm{i}$ and $4 \mathrm{a}$ as an alternative to 8d). In a majority of the cases, it appears, the competitor locates the noncommon dot in a position where it is more predictable from the other four.

\section{Stimuli and Associations}

Each of Handel and Garner's Ss responded to each of the 126 patterns, and the researchers thus obtained a matrix of 126 stimuli by 126 possible responses in which each cell entry indicated the number of Ss who made the given response to the given stimulus. Because the four or eight members of an equivalence set generally behaved the same way and often elicited one another as associates, as noted above, the researchers averaged the frequency-of-association scores over the patterns within each equivalence set for both stimuli and responses. This device reduced the data to a 23 by 23 matrix listing the mean frequency with which each of the 23 fundamentally different stimuli elicited each of the 23 fundamentally different responses. "Duplicate" associations involving rotations and reflections of the stimulus or of the response were averaged together in this matrix. Of the 21 fundamentally different cases in which a stimulus elicited a response from the same equivalence set (the cases on the diagonal in the matrix), all occurred with a mean frequency greater than unity, the value for a uniform distribution in which each pattern was used as an associate equally often to each other pattern. Of the 506 fundamentally different cases in which a stimulus elicited a response from a different equivalence set (those off the diagonal), 127 occurred with a mean frequency greater than unity.

Of these 127 stimulus-response pairs, 63 , or almost exactly half, are interpretable as associations which moved one dot in the stimulus. Because Handel and Garner reported data only for the reduced matrix, one cannot rule out the possibility that some of these associations also included a rotation or reflection of the stimulus, but it seems unlikely that many of them did. Seven of the 63 cases involve Pattern $1 \mathrm{a}$ and $1 \mathrm{~b}$, which are not altered by rotation or reflection, and there is no ambiguity about the one-dot change in these cases. Table 1 lists these 63 cases along with the lower bound of the average frequency with which each occurred. Thus the number " 1 " for the first line of the table indicates that the eight patterns in Equivalence Set $8 \mathrm{j}$ elicited as an association one of the four patterns of Set $4 \mathrm{a}$ with a mean frequency between 1 and 2 over the 8 by 4 cells involved. The number " 3 " in the third line, similarly, indicates a mean frequency between 3 and 4 for the 8 by 4 associations, in which a pattern from Set $8 \mathrm{j}$ was the stimulus and a pattern from Set $4 \mathrm{~g}$ was the response. The 63 cases in Table 1 represent between $16.7 \%$ and $28.4 \%$ of the 15,789 associations which Handel and Garner obtained. (The uncertainty about the percentage here and below is the result of their reporting only the lower bounds for the average frequencies.)

\section{Predictabilities of the Dots}

The other data in Table 1 concern the predictabilities of the figural elements. They were obtained by the present author (Bear, 1973) from Ss who were shown all possible four-dot subpatterns and asked to add to each a fifth dot "implied or suggested by those already present." The percentage of Ss who placed their dot in a given position in response to a given configuration of four dots was taken as an index of the predictability of that dot in the total five-dot pattern. The predictability scores thus obtained are reported in Fig. 1 within the dots of the patterns. The scores there have been averaged over two factors which accounted for only a small proportion of the variance in the scores: patterns within equivalence sets and, in the case of sets of Size 4, structurally equivalent locations (locations switched by rotations and reflections which leave the remainder of the pattern unchanged, such as the two corner dots in the figures of Sets $4 a$ and $4 c$ ).

As Table 1 indicates, many of the associations interpretable as one-dot changes are cases in which the response located the dot in a more predictable position, thus creating what might be called a stronger competitor of the stimulus-a pattern that is superior in competing with the stimulus for the noncommon dot. The change was to a more predictable position in 48 of the 63 cases (those with a positive number in the final column of Table 1). These account for $78.3 \%$ to $79.7 \%$ of the one-dot changes and $13.3 \%$ to $22.3 \%$ of all associations. All 10 of the equivalence sets of Size 8 have at least two stronger competitors, and 6 of the 11 sets of Size 4 have at least one. The increase in the predictability of the noncommon dot between the stimulus and the response is relatively trivial, less than 10 percentage points in 7 of the 48 cases but 50 points or more in 27 of the cases, which is over half of them.

In all but one of these 48 cases (the exception is $8 \mathrm{i}$ to $8 \mathrm{~d}$ ), it should be noted, the response pattern has not just a higher predictability for the noncommon dot but a higher mean predictability for its five elements, and 
Table 1

Associations Obtained by Handel and Garner (1966) that are Interpretable as One-Dot Changes

\begin{tabular}{|c|c|c|c|c|c|}
\hline $\begin{array}{l}\text { Stim- } \\
\text { ulus }\end{array}$ & Response & $\begin{array}{c}\text { Fre- } \\
\text { quency* }\end{array}$ & $\begin{array}{l}\text { Predicta } \\
\text { Noncom } \\
\text { in } \\
\text { Stimulus }\end{array}$ & $\begin{array}{l}\text { ability of } \\
\text { amon Dot } \\
\text { in } \\
\text { Response }\end{array}$ & $\begin{array}{l}\text { Diffe- } \\
\text { rence in } \\
\text { Predic- } \\
\text { tability }\end{array}$ \\
\hline $8 \mathrm{j}$ & $\begin{array}{l}4 \mathrm{a} \\
4 \mathrm{~h} \\
4 \mathrm{~g} \\
8 \mathrm{~b} \\
8 \mathrm{a}\end{array}$ & $\begin{array}{l}1 \\
1 \\
3 \\
1 \\
1\end{array}$ & $\begin{array}{r}1 \\
8 \\
11 \\
11 \\
11\end{array}$ & $\begin{array}{r}91 \\
61 \\
40 \\
23 \\
9\end{array}$ & $\begin{array}{r}90 \\
53 \\
29 \\
12 \\
-2\end{array}$ \\
\hline $8 \mathrm{i}$ & $\begin{array}{l}1 b \\
4 a \\
4 f \\
4 d \\
4 h \\
8 d\end{array}$ & $\begin{array}{r}10 \\
1 \\
1 \\
1 \\
1 \\
1\end{array}$ & $\begin{array}{c}1 \\
4 \\
13 \\
15 \\
15 \\
15 \text { or } 1+\end{array}$ & $\begin{array}{c}92-95 \\
91 \\
50 \\
38 \\
20 \\
17 \text { or } 3\end{array}$ & $\begin{array}{c}91-94 \\
87 \\
37 \\
23 \\
5 \\
2\end{array}$ \\
\hline $8 \mathrm{~h}$ & $\begin{array}{l}4 d \\
4 b \\
4 h \\
4 e \\
4 j \\
4 g\end{array}$ & $\begin{array}{l}2 \\
1 \\
1 \\
2 \\
1 \\
1\end{array}$ & $\begin{array}{l}0 \\
1 \\
2 \\
5 \\
5 \\
1\end{array}$ & $\begin{array}{r}91 \\
88 \\
82 \\
59 \\
16 \\
4\end{array}$ & $\begin{array}{r}91 \\
87 \\
80 \\
54 \\
11 \\
3\end{array}$ \\
\hline $8 g$ & $\begin{array}{l}4 b \\
4 e \\
8 d \\
8 b\end{array}$ & $\begin{array}{l}2 \\
2 \\
1 \\
1\end{array}$ & $\begin{array}{c}2 \text { or } 0 \dagger \\
2 \\
0 \\
2\end{array}$ & $\begin{array}{c}73 \text { or } 62 \\
19 \\
8 \\
4\end{array}$ & $\begin{array}{c}71 \text { or } 62 \\
17 \\
8 \\
2\end{array}$ \\
\hline $8 \mathrm{f}$ & $\begin{array}{l}1 \mathrm{a} \\
4 \mathrm{a} \\
4 \mathrm{~d} \\
4 \mathrm{~g} \\
8 \mathrm{a}\end{array}$ & $\begin{array}{r}13 \\
1 \\
2 \\
1 \\
1\end{array}$ & $\begin{array}{c}2 \\
1 \\
1 \\
16 \\
1 \text { or } 2 \dagger\end{array}$ & $\begin{array}{c}93-98 \\
54 \\
25 \\
40 \\
1 \text { or } 0\end{array}$ & $\begin{array}{c}91-96 \\
53 \\
24 \\
24 \\
0 \text { or }-2\end{array}$ \\
\hline $8 e$ & $\begin{array}{l}4 a \\
4 b \\
8 b\end{array}$ & $\begin{array}{l}1 \\
2 \\
1\end{array}$ & $\begin{array}{l}1 \\
2 \\
2\end{array}$ & $\begin{array}{r}96 \\
73 \\
4\end{array}$ & $\begin{array}{r}95 \\
71 \\
2\end{array}$ \\
\hline $8 d$ & $\begin{array}{l}1 b \\
4 a \\
8 b\end{array}$ & $\begin{array}{l}4 \\
3 \\
1\end{array}$ & $\begin{array}{l}3 \\
2 \\
2\end{array}$ & $\begin{array}{c}92-95 \\
93 \\
2\end{array}$ & $\begin{array}{c}89-92 \\
91 \\
0\end{array}$ \\
\hline $8 c$ & $\begin{array}{l}4 a \\
4 d \\
4 f\end{array}$ & $\begin{array}{l}2 \\
1 \\
1\end{array}$ & $\begin{array}{l}1 \\
4 \\
1\end{array}$ & $\begin{array}{l}96 \\
91 \\
15\end{array}$ & $\begin{array}{l}95 \\
87 \\
14\end{array}$ \\
\hline $8 b$ & $\begin{array}{l}4 a \\
4 h \\
4 b \\
4 e\end{array}$ & $\begin{array}{l}1 \\
1 \\
1 \\
1\end{array}$ & $\begin{array}{l}2 \\
8 \\
4 \\
4\end{array}$ & $\begin{array}{l}93 \\
79 \\
73 \\
19\end{array}$ & $\begin{array}{l}91 \\
71 \\
69 \\
15\end{array}$ \\
\hline $8 a$ & $\begin{array}{l}1 \mathrm{a} \\
4 \mathrm{~h}\end{array}$ & $\begin{array}{l}3 \\
1\end{array}$ & $\begin{array}{l}0 \\
2\end{array}$ & $\begin{array}{c}93-98 \\
79\end{array}$ & $\begin{array}{c}93-98 \\
77\end{array}$ \\
\hline $4 k$ & $\begin{array}{l}4 b \\
4 c \\
4 g\end{array}$ & $\begin{array}{l}3 \\
1 \\
1\end{array}$ & $\begin{array}{r}5 \\
13 \\
5\end{array}$ & $\begin{array}{r}88 \\
50 \\
4\end{array}$ & $\begin{array}{r}83 \\
37 \\
-1\end{array}$ \\
\hline $4 j$ & $\begin{array}{l}1 \mathrm{a} \\
8 \mathrm{a}\end{array}$ & $\begin{array}{l}8 \\
1\end{array}$ & $\begin{array}{r}1 \\
16\end{array}$ & $\begin{array}{l}97 \\
13\end{array}$ & $\begin{array}{r}96 \\
-3\end{array}$ \\
\hline $4 i$ & $1 b$ & 9 & 1 & 97 & 96 \\
\hline $4 h$ & $8 b$ & 2 & 79 & 8 & -71 \\
\hline $4 \mathrm{~g}$ & $\begin{array}{l}4 b \\
4 d \\
8 b\end{array}$ & $\begin{array}{l}1 \\
2 \\
1\end{array}$ & $\begin{array}{r}4 \\
10 \\
40\end{array}$ & $\begin{array}{l}88 \\
38 \\
23\end{array}$ & $\begin{array}{r}84 \\
28 \\
-17\end{array}$ \\
\hline $4 f$ & $\begin{array}{l}4 c \\
4 a \\
4 d\end{array}$ & $\begin{array}{l}1 \\
2 \\
1\end{array}$ & $\begin{array}{l}15 \\
19 \\
19\end{array}$ & $\begin{array}{l}65 \\
54 \\
25\end{array}$ & $\begin{array}{r}50 \\
35 \\
6\end{array}$ \\
\hline $4 e$ & $8 b$ & 1 & 19 & 4 & -15 \\
\hline
\end{tabular}

Table 1 (Continued)

$\begin{array}{rrrrrr} & 4 \mathrm{a} & 1 & 25 & 54 & 29 \\ 4 \mathrm{~d} & 4 \mathrm{f} & 2 & 25 & 19 & -6 \\ & 4 \mathrm{~g} & 1 & 38 & 10 & -28 \\ & 4 \mathrm{j} & 1 & 50 & 10 & -40 \\ 4 \mathrm{c} & 4 \mathrm{e} & 2 & 65 & 17 & -48 \\ & 4 \mathrm{f} & 3 & 65 & 15 & -50 \\ 4 \mathrm{~b} & 4 \mathrm{~g} & 1 & 88 & 4 & -84 \\ 4 \mathrm{a} & - & & & & \\ 1 \mathrm{~b} & 4 \mathrm{i} & 2 & 97 & 1 & -96 \\ 1 \mathrm{a} & - & & & & \end{array}$

Note-Patterns are labeled as in the Handel and Garner study and as in Fig. 1.

* Lower bound of average frequency with which the stimulusresponse pair occurred. A verage was taken over all members of the equivalence set of stimuli and over all members of the equivalence set of responses. Value would be unity if each pattern were used equally often as an association to each other pattern.

TMoving either of two dots in the stimulus pattern converts it to a response pattern in the indicated equivalence set.

mean predictability of elements (which is indicated in Fig. 1 on the bottom line below each pattern) is closely related to number of implicit aiternatives and to difficulty of encoding. The correlations are $-.93,-.78$, and .85 with the number-of-alternatives measures and $-.79,-.85,-.87$, and -.87 with the difficulty-ofencoding measures; all values are in the "right" direction. Thus the trend toward a stronger competitor in which the noncommon dot is more predictable from the remaining elements is also a trend toward a pattern whose other elements are more predictable one from the others and a trend toward a pattern which has fewer implicit alternatives and is more easily encoded. This trend accords with the Gestaltists' law of Prügnanz, which asserts a tendency for psychological processes to move toward configurations of elements which are figurally "good," i.e., simple, or easy to encode, and well organized, or composed of predictable elements. Ss' judgments of "pattern goodness" for the dot figures, in fact, correlate highly in the required directions with each of the other measures described above. Judgments collected by Garner and Clement, by Handel and Garner, and by Clement (1964) are closely related to each other $(r=.98-.99)$ and yield a set of coefficients for the other measures that range in absolute value from .68 to .95 , with a median of $.89 .^{3}$

There are some exceptions, of course, to the trend toward a stronger competitor with an increase in the predictability of the noncommon dot. As Table 1 shows, an increase failed to occur in 15 of the 63 cases of a one-dot change, and in 4 of these cases there was a decline of 50 percentage points or more. Some of the 15 exceptional cases, though, may be artifacts of Handel and Garner's procedure. Their Ss may have made some "unnatural" responses because they were not permitted to use a pattern as its own association or because they were bored with the task. 


\section{SCHEMA-PLUS-CORRECTION ENCODING}

The data from which Table 1 was derived were not specifically collected to provide information on the encoding of the dot patterns, and the only firm conclusion which Table 1 warrants is just what it shows: The subset of alternatives which a $S$ (on Garner's hypothesis) infers when presented with a dot pattern includes not just the rotations and reflections of the pattern but in many cases one or more other patterns which differ from the stimulus in the position of a single dot and that generally locate that dot in a position where it is more predictable from the other four. Table 1 does suggest, though, that certain of the patterns are encoded as a "schema plus correction," at least at some stage of the processing of the pattern in at least some tasks, and a brief discussion of this matter may have some heuristic value.

The possibility of schema-plus-correction encoding was suggested some years ago (Woodworth, 1938, Chapter 4), but it seems to have been generally ignored in recent treatments of encoding. The theories of the processing of sequential elements reviewed by Simon (1972), for example [Payne's (1966) is another such theory], and Vitz and Todd's (1971) model for the perception of geometric figures do not permit this means of representing a pattern. The one-dot changes which may constitute perhaps a fifth of Handel and Garner's associations, though, are plausibly interpreted as cases in which the $\mathbf{S}$ dropped the correction to a stimulus pattern encoded as a schema plus correction, thereby revealing the schema in the pattern which he drew as a response.

On this interpretation, the patterns which occur as responses in Table 1 represent not just implicit alternatives to the stimuli listed in the table but also schemas which the Ss used in encoding the stimuli. The equivalence sets to which the responses belong are la, $1 \mathrm{~b}, 4 \mathrm{a}$ through $4 \mathrm{j}$, and $8 \mathrm{a}, 8 \mathrm{~b}$, and $8 \mathrm{~d}$. Most of the schemas thus seem to be simple, well organized "good" figures, "natural prototypes" perhaps (Rosch, 1973), easily encoded themselves when they appear as stimuli and composed of dots which are more or less predictable one from the others, as shown in Fig. 1. The dot whose position differs between the stimulus and the schema, in particular, is generally located in a more predictable position in the schema, and the schema is thus generally more "plausible" than the stimulus, given the four dots they have in common (cf. Bruner, 1957).

The concept of schema-plus-correction encoding raises a number of questions which may be worth pursuing. For example, how is the schema selected? The generally greater predictability of the noncommon dot in Handel and Garner's one-dot-different associations suggests the possibility that the selection is made by extrapolating the schema from a sample of the stimulus, and if this is so, the problem then arises of how the sample is selected. A related question is how different from the stimulus the schema can be. Associations such as 1a to $4 \mathrm{a}$ and $4 \mathrm{e}$ to $4 \mathrm{i}$ suggest that not only one-element but also two-element differences are possible for the dot patterns. A third question is why a stimulus such as $8 \mathrm{~h}$, which is apparently susceptible to several different schema-plus-correction encodings, should be more difficult to encode than a stimulus susceptible only to one or to none at all, in keeping with the correlation between number of implicit alternatives and difficulty of encoding. The most basic question, of course, is whether the implicit alternatives play a causal role in the encoding process or whether they are just a by-product, as Lappin has argued (1971; Lappin, Snyder, \& Blackburn, 1971).

\section{REFERENCES}

Bear, G. Redundancy, predictability of figural elements, and figural goodness. (Doctoral dissertation, Yale University) Ann Arbor, Mich: University Microfilms, 1970. No.70-25, 236.

Bear, G. Figural goodness and the predictability of figural elements. Perception \& Psychophysics, 1973, 13, 32-40.

Bruner, J. S. On perceptual readiness. Psychological Review, $1957,64,123-152$.

Clement, D. E. Uncertainty and latency of verbal naming responses as correlates of pattern goodness. Journal of Verbal Learning \& Verbal Behavior, 1964, 3, 150-157.

Clement, D. E. Paired-associate learning as a correlate of pattern goodness. Journal of Verbal Learning \& Verbal Behavior, $1967,6,112-116$.

Clement, D. E., Guenther, Z. C., \& Sistrunk, F. Incertidumbre y percepcion de patrones: Reseĩa y perspectiva. Revista Latinoamericana de Psicologia, 1972, 4, 177-188.

Clement, D. E., \& Sistrunk, F. Judgments of pattern goodness and pattern preference as functions of age and pattern uncertainty. Developmental Psychology, 1971, 5, 389-394.

Clement, D. E., Sistrunk, F., \& Guenther, Z. C. Pattern perception among Brazilians as a function of pattern uncertainty and age. Journal of Cross-Cultural Psychology, 1970, 1, 305-313.

Clement, D. E., \& Weiman, C. F. R. Instructions, strategies, and pattern uncertainty in a visual discrimination task. Perception \& Psychophysics, 1970, 7, 333-336.

Garner, W. R. Uncertainty and structure as psychological concepts. New York: Wiley, 1962.

Garner, W. R. To perceive is to know. American Psychologist, $1966,21,11-19$.

Garner, W. R., \& Clement, D. E. Goodness of pattern and pattern uncertainty. Journal of Verbal Learning \& Verbal Behavior, 1963, 2, 446-452.

Glanzer, M., Taub, T., \& Murphy, R. An evaluation of three theories of figural organization. American Journal of Psychology, 1968, 81, 53-66.

Handel, S., \& Buffardi, L. Pattern perception: Integrating information presented in two modalities. Science, 1968, 162, 1026-1028.

Handel, S., \& Garner, W. R. The structure of visual pattern associates and pattern goodness. Perception \& Psychophysics, 1966, 1, 33-38.

Lappin, J. S. Transformation-invariant cues in the recognition of simple visual patterns. Perception \& Psychophysics, 1971, 10, $367-370$.

Lappin, J. S., Snyder, C. R., \& Blackburn, C. The encoding of perceptual information in the organization of individual stimulus patterns. Perception \& Psychophysics, 1971, 10, 123-128. 
Payne, B. The relationship between a measure of organization for visual patterns and their judged complexity, Journal of Verbal Learning \& Verbal Behavior, 1966, 5, 338-343.

Prokhovnik, S. J. Pattern variants on a square field. Psychometrika, 1959, 24, 329-341.

Rosch, E. H. Natural categories. Cognitive Psychology, 1973, 4, 328-350.

Royer, F. L., \& Garner, W. R. Response uncertainty and perceptual difficulty of auditory temporal patterns. Perception \& Psychophysics, 1966, 1, 41-47.

Simon, H. A. Complexity and the representation of patterned sequences of symbols. Psychological Review, 1972, 79, 369-382.

Vitz, P. C., \& Todd, T. C. A model of the perception of simple geometric figures. Psychological Review, 1971, 78, 207-228.

Woodworth, R. S. Experimental psychology. New York: Holt, 1938.

\section{NOTES}

1. Garner has referred to the stimuli in the subset which he hypothesizes the $S$ to infer as "equivalents" to the stimulus as well as "alternatives" to it. The word "alternatives" is used here to avoid confusion of the hypothetical subjective subset with the objectively defined equivalence set of rotations and reflections. Another term employed in reference to the inferred stimuli is the "uncertainty" of the presented stimulus.

2. Garner and Clement reported nothing about which particular patterns were judged similar to which others, except that "most of the Ss kept the reflection and rotation equivalence groups intact [p. 488]." Their study thus does not speak to the present issue.

3. The judgments of pattern goodness were obtained to test Garner's (1962) hypothesis that a good figure has relatively few implicit alternatives. As the present review indicates, the hypothesis has been strongly confirmed.
(Received for publication October 1, 1973; accepted October 22, 1973.) 